\title{
Role of the small RNA RyhB in the Fur regulon in mediating the capsular polysaccharide biosynthesis and iron acquisition systems in Klebsiella pneumoniae
}

Su-Hua Huang ${ }^{1 \dagger}$, Chien-Kuo Wang ${ }^{1 \dagger}$, Hwei-Ling Peng ${ }^{2}$, Chien-Chen Wu ${ }^{2}$, Ying-Tsong Chen ${ }^{3,4,5}$, Yi-Ming Hong ${ }^{6}$ and Ching-Ting Lin ${ }^{6^{*}}$

\begin{abstract}
Background: The capsular polysaccharide (CPS) and iron acquisition systems are important determinants of Klebsiella pneumoniae infections, and we have previously reported that the ferric uptake repressor (Fur) can play dual role in iron acquisition and CPS biosynthesis. In many bacteria, Fur negatively controls the transcription of the small non-coding RNA RyhB to modulate cellular functions and virulence. However, in K. pneumoniae, the role played by RyhB in the Fur regulon has not been characterised. This study investigated Fur regulation of ryhB transcription and the functional role of RyhB in K. pneumoniae.

Results: Deletion of fur from K. pneumoniae increased the transcription of ryhB; the electric mobility shift assay and the Fur-titration assay revealed that Fur could bind to the promoter region of ryhB, suggesting that Fur directly represses ryhB transcription. Additionally, in a $\triangle$ fur strain with elevated CPS production, deletion of ryhB obviously reduced CPS production. The following promoter-reporter assay and quantitative real-time PCR of cps genes verified that RyhB activated orf1 and orf16 transcription to elevate CPS production. However, deletion of ryhB did not affect the mRNA levels of $r C S A$, rmpA, or $r m p A 2$. These results imply that Fur represses the transcription of ryhB to mediate the biosynthesis of CPS, which is independent of RcsA, RmpA, and RmpA2. In addition, the $\triangle$ fur strain's high level of serum resistance was attenuated by the deletion of ryhB, indicating that RyhB plays a positive role in protecting the bacterium from serum killing. Finally, deletion of ryhB in $\Delta$ fur reduced the expression of several genes corresponding to 3 iron acquisition systems in K. pneumoniae, and resulted in reduced siderophore production.
\end{abstract}

Conclusions: The regulation and functional role of RyhB in K. pneumoniae is characterized in this study. RyhB participates in Fur regulon to modulate the bacterial CPS biosynthesis and iron acquisition systems in $K$. pneumoniae.

Keywords: RyhB, Fur, Capsular polysaccharide, Iron acquisition system, Klebsiella pneumoniae

\footnotetext{
* Correspondence: gingting@mail.cmu.edu.tw

'Equal contributors

${ }^{6}$ School of Chinese Medicine, China Medical University, Taichung 40402,

Taiwan

Full list of author information is available at the end of the article
} 


\section{Background}

Klebsiella pneumoniae, a member of Enterobacteriaceae, is a rod-shaped gram-negative opportunistic pathogen. A common cause of nosocomial infection, it is also found in various community-acquired infections, including bacteraemia, septicaemia, and urinary tract and respiratory infections, particularly in immunocompromised patients [1-4]. In Asian countries, especially Taiwan and Korea, $K$. pneumoniae is the predominant pathogen found in pyogenic liver abscess in diabetic patients $[2,3,5]$. The rapid development of antimicrobial resistance in K. pneumoniae has further troubled the clinical choices for treatments $[6,7]$. Studies of the pathogenic mechanisms of K. pneumoniae are, therefore, essential in identifying new targets for the development of antibacterial agents.

Multiple virulence factors have been identified to be involved in $K$. pneumoniae infection, which include capsular polysaccharide (CPS), lipopolysaccharides, fimbriae, iron-acquisition system, and antibiotic resistance. Among these factors, CPS is probably considered the major determinants of pathogenesis. The pyogenic liver abscess isolates often carry heavy CPS that could protect the bacteria from phagocytosis and killing by serum factors [8,9]. Apart from the antiphagocytic function, Klebsiella CPS also helps the bacterial colonization and biofilm formation at the infection sites [10-12]. The capsular serotypes of $K$. pneumoniae have been classified as more than 77 recognized capsular antigens $[13,14]$. In Taiwan, a high prevalence of $\mathrm{K} 1$ and $\mathrm{K} 2$ serotypes of $K$. pneumoniae was documented in liver abscess of diabetes mellitus patients [15]. The cps gene clusters that are responsible for the synthesis of different serotypes of CPS have been determined [16]. The K2 cps gene cluster of $K$. pneumoniae Chedid contains a total number of 19 open reading frames (ORFs) organized into three transcription units, orf1-2, orf3-15, and orf16-17 [16]. In the previous studies, numerous regulatory systems were demonstrated to control the biosynthesis of CPS via regulating the cps transcriptions in $K$. pneumoniae, such as the Rcs system, RmpA, RmpA2, KvhR, KvgAS, and KvhAS [1720]. Among these, ferric uptake regulator (Fur) represses the gene expression of $\operatorname{rcs} A, \operatorname{rmp} A$, and rmpA2 to decrease CPS biosynthesis [21,22]. Therefore, overlapping regulons governed the regulation of these assorted virulence genes in response to numerous stress conditions.

Bacterial cells are constantly challenged by various environmental stresses from their natural habitats. Similar to many gastrointestinal (GI) pathogens, K. pneumoniae faces several challenges during infection and colonisation of the human body. These include gastric acid, the immune system, and a limited supply of oxygen and nutrients [23,24]. Among these, the concentration of iron in the environment is critical for the control of cellular metabolism. Limitation of iron abolishes bacterial growth, but high intracellular concentrations of iron may damage bacteria because of the formation of undesired reactive oxygen species (ROS). Iron homeostasis maintained by the transport, storage, and metabolism of iron is tightly controlled by Fur in many gram-negative bacteria [25-27]. To regulate gene transcription, Fur protein functions as a dimer with $\mathrm{Fe}^{2+}$ as a cofactor to bind to a 19-bp consensus sequence, called the Fur box (GATAATGATwATCATTATC; $\mathrm{w}=\mathrm{A}$ or $\mathrm{T}$ ), in the promoters of downstream genes [28]. In several gramnegative pathogens, Fur represses the expression of genes involved in iron homeostasis and in the regulation of multiple cellular functions such as oxidative stress, energy metabolism, acid tolerance, and virulence gene production [29-32]. In K. pneumoniae, Fur plays a dual role in controlling CPS biosynthesis and iron acquisition [21]. Recently, we also found that type 3 fimbriae expression and bacterial biofilm formation were also controlled by Fur and iron availability [33]. Therefore, the regulatory mechanism of Fur in control of multiple cellular function and virulence factors in $K$. pneumoniae needs to be further investigated.

Although Fur typically acts as a repressor, it also functions as a transcriptional activator for the gene expression such as $a c n A$, fumA, and $s d h C D A B$ (tricarboxylic acid [TCA] cycle enzymes), bfr and $f t n A$ (iron storage), and $\operatorname{sod} B$ (iron superoxide dismutase [FeSOD]) [34-38]. However, positive regulation by Fur is often indirect, mediated by Fur-dependent repression of a small non-coding RNA (sRNA), RyhB [39]. RyhB negatively regulates gene expression by base pairing with mRNAs to trigger their degradation via RNase E and RNase III [40]. In many bacteria, RyhB participates in Fur-mediated positive regulation of various important cellular functions, including TCA cycle activity, resistance to oxidative stress, and iron homeostasis in Escherichia coli and Vibrio cholerae [35,39,41-43]; biofilm formation in $V$. cholerae [44]; and virulence in Shigella dysenteriae [45]. In E. coli, RyhB has been demonstrated to directly regulate more than 18 transcripts, encoding a total of 56 proteins, most of them involved in iron metabolism [35]. Although the significance of RyhB has been demonstrated in different species, to date, the regulatory relationship of RyhB and Fur, and functionality of RyhB in $K$. pneumoniae has not been studied.

In this study, the regulatory role of Fur in $r y h B$ expression in $K$. pneumoniae was investigated. A ryhB-deletion mutant in wild type (WT) and $\Delta$ fur strains and the induced expression of $r y h B$ in WT were generated to demonstrate the role of RyhB in mediating CPS biosynthesis and iron acquisition systems. 


\section{Results}

Fur directly represses ryhB expression in $K$. pneumoniae To determine whether $K$. pneumoniae $r y h B$ is regulated by Fur, a LacZ reporter system was used. The $r y h B$ promoter was cloned into the upstream region of a promoterless $l a c Z$ gene in placZ15. The resulting plasmid pRyhB15 was then introduced into $K$. pneumoniae CG43S3 $\Delta l a c Z$ and $\Delta l a c Z \Delta$ fur. The bacterial $\beta$-galactosidase activity was measured to assess the expression level of $r y h B$. As shown in Figure 1A, the expression of $r y h B$ was higher in $\Delta l a c Z \Delta$ fur than $\Delta l a c Z$. Introduction of the complement plasmid pfur, but not the empty vector control (pRK415), into $\Delta l a c Z \Delta$ fur restored the Fur-deletion effect. Moreover, addition of the iron chelator 2, 2-dipyridyl (Dip) to the growth medium increased $r y h B$ promoter activity, suggesting that a Fur-Fe(II) complex influences $r y h B$ expression. To verify that Fur directly regulates the expression of $r y h B$, an electrophoretic mobility shift assay (EMSA) was performed. As shown in Figure 1B, purified recombinant His $_{6}$-Fur protein was able to bind the upstream region of $\operatorname{ryh} B\left(\mathrm{P}_{r y h B}\right)$, but not the $\mathrm{P}_{r y h B^{*}}$ fragment, whose putative Fur-box was deleted. In addition, the binding ability was abolished by the addition of $200 \mu \mathrm{M}$ EDTA to the reaction mixture (data not shown). Furthermore, E. coli H1717,

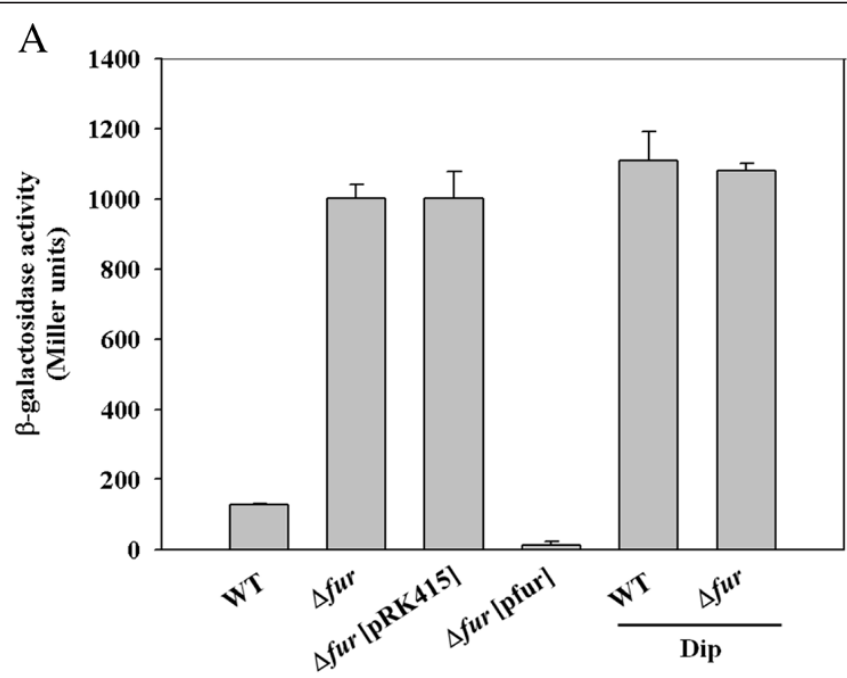

B His6::Fur $(\mathrm{nM})$
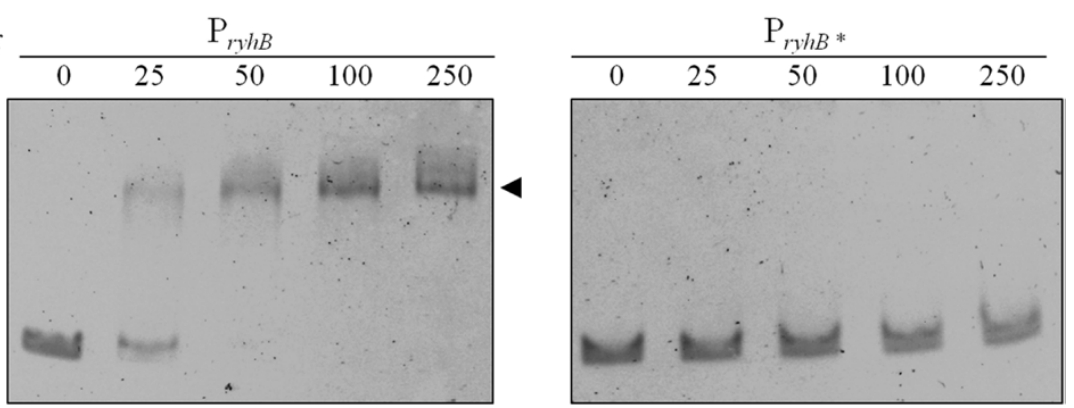

C
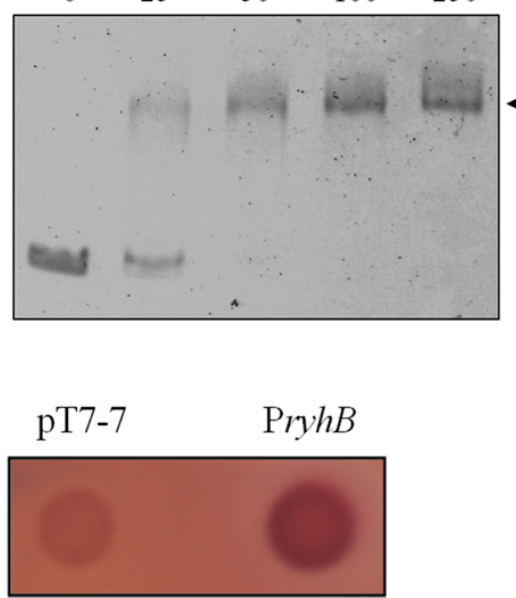

Figure 1 Fur directly represses the expression of $r y h B$. (A) The $\beta$-galactosidase activities of the $K$. pneumoniae CG43S3 $\Delta$ lacZ strain and the

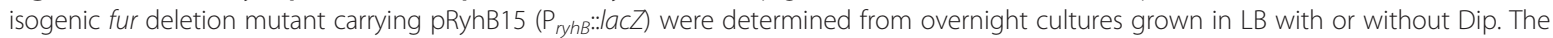
plasmids pRK415 (vector control) and pfur were introduced into $\Delta$ fur to observe the complement effect. The average of triplicate experiments is shown. Error bars indicate standard deviations. (B) EMSA of the recombinant His $6:$ Fur and the ryhB promoter regions, as indicated in the margin. DNA was incubated with an increasing amount of $\mathrm{His}_{6}:$ :Fur for $30 \mathrm{~min}$, and then loaded onto a $5 \%$ non-denaturing polyacrylamide gel. The gel was stained with SYBR Green EMSA stain and photographed. $P_{r y h B}{ }^{*}$ indicates deletion of the fur box in $P_{r y h B}$. (C) Assessment of the binding of Fur to the ryhB promoter by using the FURTA. E. coli H1717 strains carrying the vector control, pT7-7, or the P1 region harboured on pT7-7 are indicated. A red colony $\left(\operatorname{Lac}^{+}\right)$is considered to have a FURTA-positive phenotype. 
when harbouring a plasmid containing $K$. pneumoniae $\mathrm{P}_{r y h B}$, also showed a Fur titration assay (FURTA)-positive phenotype (Figure $1 \mathrm{C}$ ). The results suggest that, in an iron dependent manner, Fur suppresses ryhB promoter activity in $K$. pneumoniae by direct interaction with the Fur-box region upstream of $r y h B$.

\section{RyhB activates CPS biosynthesis}

In K. pneumoniae CG43, we found that the deletion of fur resulted in elevated CPS production [21,22]. To investigate if RyhB participates in Fur-regulated CPS biosynthesis, the CPS amount was assessed using measuring glucuronic acid content, which served as an indicator for Klebsiella K2 CPS [46], in K. pneumoniae strains, including WT, $\Delta r y h B, \Delta f u r$, and $\Delta f u r \Delta r y h B$, was quantified. As shown in Figure $2 \mathrm{~A}$, although the deletion of $r y h B$ alone did not change on the amount of K2 CPS production, the elevated CPS amount in $\triangle$ fur cells was abolished by the deletion of $r y h B$ when the bacteria were grown in LB medium. The result indicates that Fur regulates the expression of RyhB to repress CPS biosynthesis. To confirm the RyhB expression could activate the CPS biosynthesis, the effect of RyhB induction on CPS amount was determined using an IPTG-inducible vector, pETQ. As shown in Figure 2B, the induced expression of ryhB in K. pneumoniae CG43 increased CPS production, which confirms that RyhB positively regulates CPS biosynthesis.

\section{RyhB increased the transcriptional level of the K2 cps gene cluster}

To investigate whether RyhB affects the expression of the three cps gene clusters, the mRNA levels of orf1, orf3, and orf16 in $\Delta$ fur and $\Delta f u r \Delta r y h B$ strains were measured by quantitative real-time PCR (qRT-PCR). As shown in Figure 3A, compared to the mRNA levels in the $\Delta$ fur strain, the mRNA levels of orf1 and orf16 were apparent decreased in the $\Delta f u r \Delta r y h B$ strain, and that of orf3 also had a slight reduction in the $\Delta f u r \Delta r y h B$ strain. The result suggests that overexpression of RyhB activated the cps gene expression. To confirm our hypothesis, the effect of $r y h B$ induction on the mRNA levels of orf1, orf3, and orf16 was tested using an IPTG-inducible vector, pETQ. As shown in Figure 3B, the mRNA levels of orf1 and orf16 were higher in the pETQ-ryhB strain with IPTG induction than the pETQ mock strain, while no significant difference in orf3 expression was observed. To further investigate whether RyhB acts as a transcriptional activator for the promoter activity of orf1, orf3,

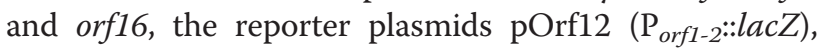

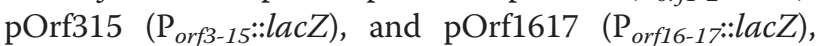
each carrying a lac $Z$ reporter gene transcriptionally fused to the putative promoter region of the $\mathrm{K} 2$ cps gene cluster [17], were used to transform the $K$. pneumoniae strains CG43S3 $\Delta l a c Z \Delta$ fur and $\Delta l a c Z \Delta f u r \Delta r y h B$. The promoter activity measurements shown in Figure $3 C$ revealed that the deletion of $r y h B$ in $\Delta l a c Z \Delta$ fur reduced activity of $\mathrm{P}_{\text {orf1-2 }}:$ lac $Z$ by at least $50 \%$, while no obvious change was detected in the activity of $\mathrm{P}_{\text {orf3-16::lacZ. The }}$ activity of $\mathrm{P}_{\text {orf } 16-17:: l a c Z}$ was reduced by more than $75 \%$ in $\Delta l a c Z \Delta f u r \Delta r y h B$ as compared to the $\Delta l a c Z \Delta f u r$ strain. These results imply that RyhB enhances CPS biosynthesis in $K$. pneumoniae by boosting the transcriptional level of the orf1 and orf16 gene clusters.

\section{RyhB does not affect the $r \operatorname{cs} A, r m p A 2$, and $r m p A$ mRNA expression level}

In previous studies, $K$. pneumoniae Fur was found to repress the expression of genes encoding the cps
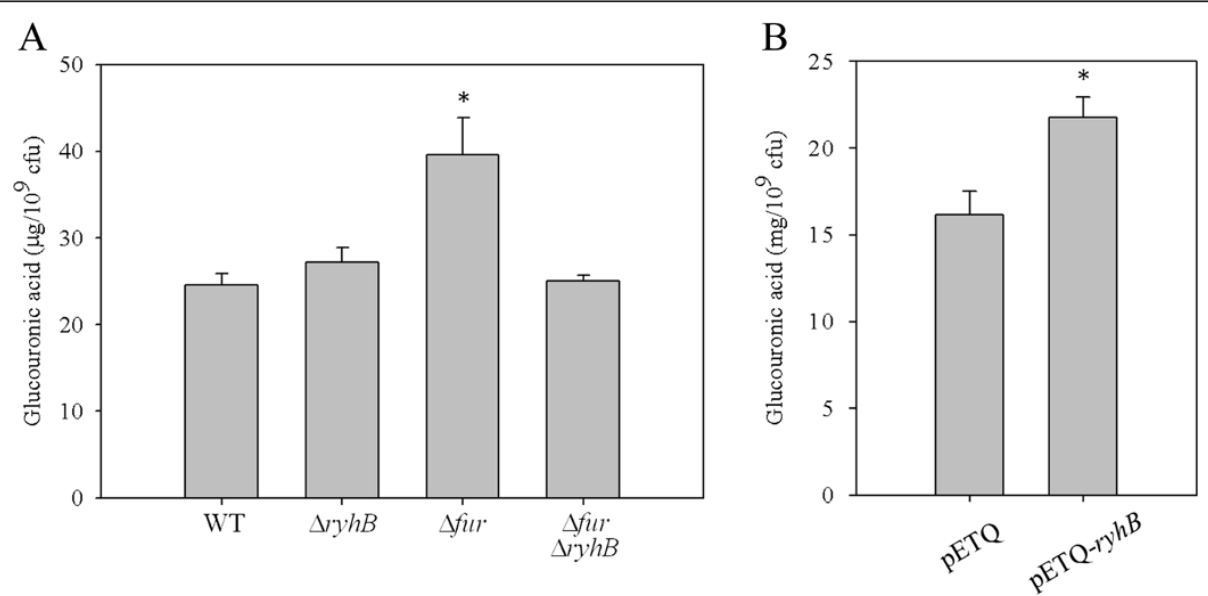

Figure 2 RyhB activates CPS biosynthesis. (A) Comparison of CPS levels in WT, $\Delta r y h B, \Delta f u r$, and $\Delta f u r \Delta r y h B$ strains. Bacterial strains were grown in $L B$ medium at $37^{\circ} \mathrm{C}$ with agitation. After $16 \mathrm{~h}$ of growth, the bacterial glucuronic acid content was determined. ${ }^{*}, P<0.001$ compared with WT. (B) WT strains carrying the vector control (pETQ) or pETQ-ryhB were grown in LB with $100 \mu \mathrm{M} \mathrm{IPTG}$ to induce ryhB expression. ${ }^{*}, P<0.001$ compared with WT strains carrying PETQ. 

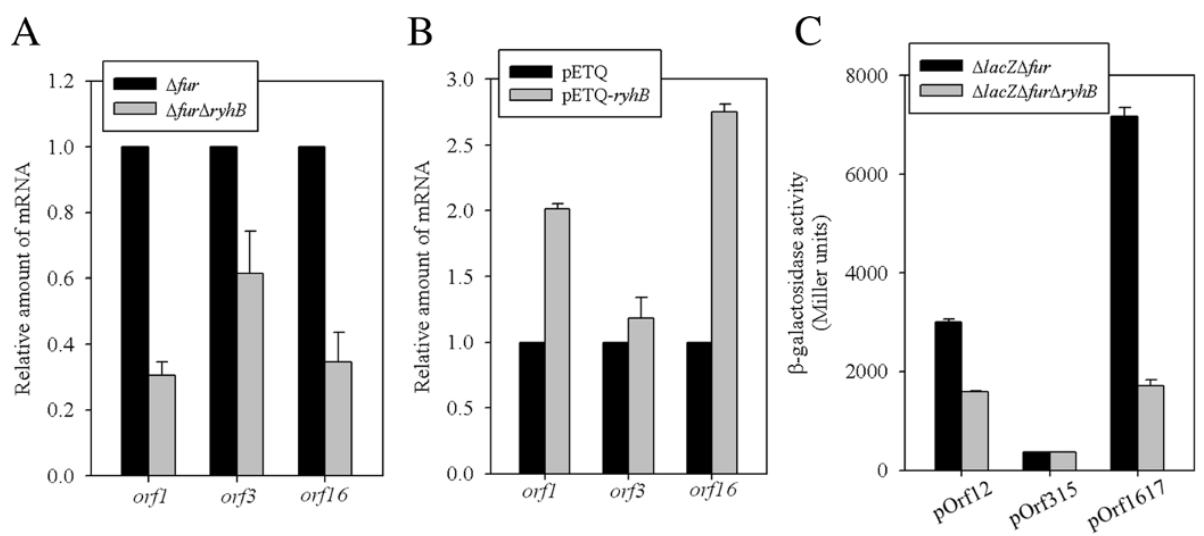

Figure 3 RyhB activates the transcriptional level of the orf1 and orf16. (A) qRT-PCR analyses of the expression of the K2 cps genes (orf1, orf3, and orf16) were measured in $\Delta$ fur and $\Delta$ fur $\Delta r y h B$ strains. (B) WT strain carrying the IPTG inducible vector pETQ and pETQ-ryhB in response to $100 \mu \mathrm{M}$ IPTG. (C) The $\beta$-galactosidase activities of $K$. pneumoniae CG4353 $\Delta / a c Z \Delta$ fur and $\Delta / a c Z \Delta$ fur $\Delta r y h B$ carrying the reporter plasmid pOrf12

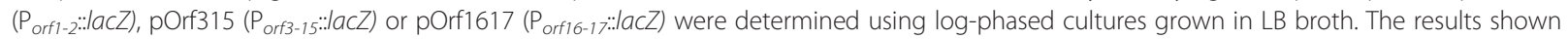
are an average of triplicate samples. Error bars indicate standard deviations.

regulatory proteins RcsA, RmpA, and RmpA2 [21,22]. To investigate whether RyhB affects the expression of $\operatorname{rcs} A, \operatorname{rmp} A$, and $\operatorname{rmp} A 2$ to increase the orf 1 and $\operatorname{orf16}$ transcripts, the mRNA levels were measured by qRTPCR after inducing the expression of ryhB in WT. However, qRT-PCR results did not show a significant effect of $r y h B$ on the mRNA levels of $r m p A, \operatorname{rmp} A 2$, and $r c s A$ (Data not shown), suggesting that the activation of RyhB on the orf1 and orf16 expression is not via RmpA, RmpA2, and RcsA.

\section{Deletion of ryhB attenuated the higher serum resistance in $\Delta$ fur strain}

In addition to the roles played by RyhB and Fur in regulating the CPS amount, we suggest that RyhB and Fur may also affect the ability of the strain to resist the bactericidal effects of serum. In a human serum resistance assay, we found that the deletion of fur in WT increased the survival rate in treatment with $75 \%$ normal human serum from $63.3 \%$ to $87.9 \%$ (Figure 4). However, the deletion of $r y h B$ in WT had no apparent effect on the survival rate on treatment with $75 \%$ serum, and the higher serum resistance in $\Delta$ fur cells was abolished by the deletion of $r y h B$. This result indicates that RyhB may participate with Fur in regulating serum resistance in $K$. pneumoniae.

The regulatory role of RyhB in iron-acquisition systems To assess whether RyhB affects iron-acquisition in $K$. pneumoniae, the Chrome azurol S (CAS) assay was used to measure siderophore secretions in $\Delta f u r$ and $\Delta$ fur$\triangle r y h B$ strains (Figure 5). When bacteria were grown in M9 minimal medium ( $\sim \mu \mathrm{M}$ iron) to mimic ironlimited condition, the deletion of $r y h B$ in $\Delta$ fur reduced the formation of the orange halo. However, this change was not observed when bacteria were grown in LB medium ( $18 \mu \mathrm{M}$ iron). Compared to M9 minimal medium contains $\sim 2 \mu \mathrm{M}$ iron, LB medium is considered an iron-repletion medium. Under iron-repletion, Fur is able to exert its repression on $r y h B$ transcription. Thus, $r y h B$-deletion effect is difficult to observed under the growth condition that $r y h B$ is poorly expressed. Our results suggest that in the regulation of iron-acquisition systems, RyhB plays a role downstream of Fur in $K$. pneumoniae under iron-limiting conditions.

To investigate the effects on downstream targets of RyhB in iron-acquisition regulons, the expression of genes corresponding to the eight putative ironacquisition systems in $K$. pneumoniae CG43 was measured in $\Delta$ fur and $\Delta f u r \Delta r y h B$ by qRT-PCR (Table 1 ). In

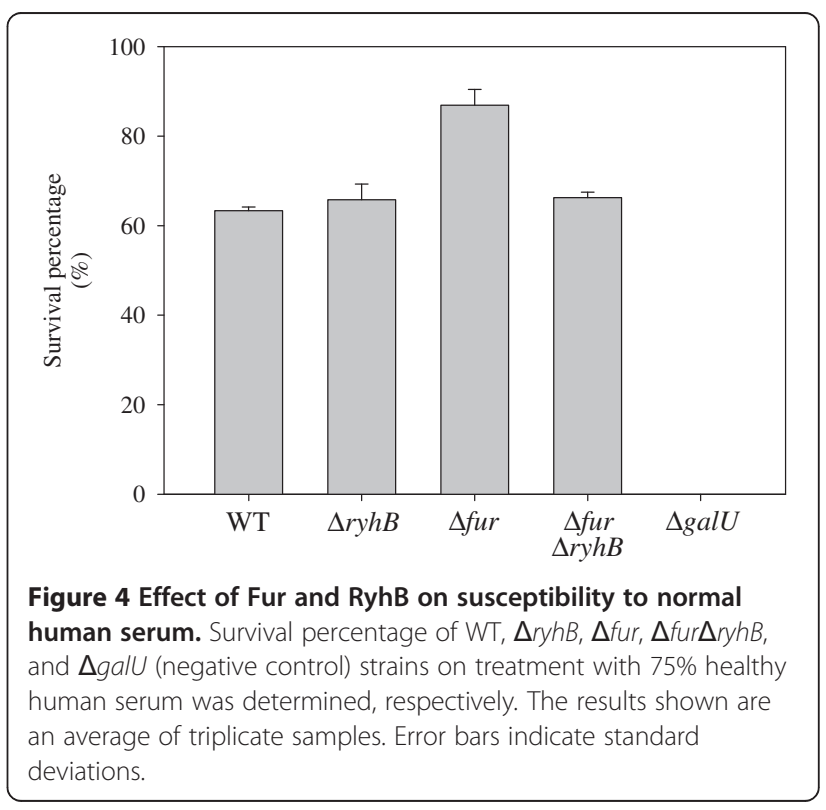




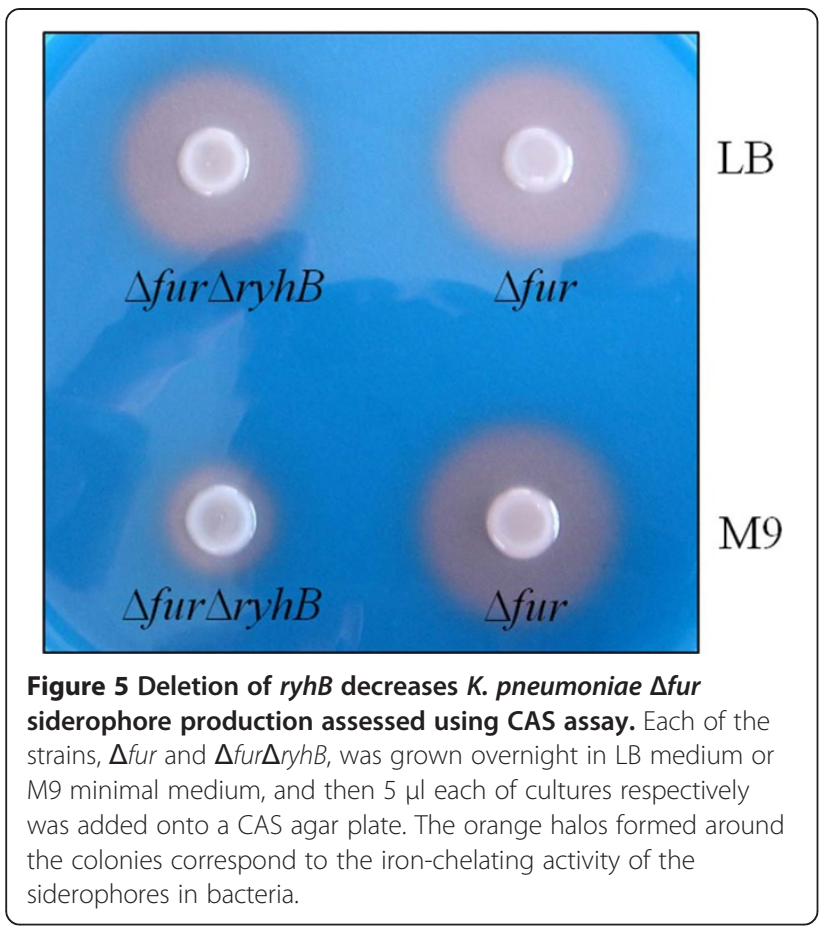

M9 minimal medium, the expression of genes (iucA, $f e p A, f e p B$, ent $C$, $f e c A$, and $f e c E$ ) corresponding to three iron-acquisition systems (aerobactin, enterobactin, and ferric citrate) was decreased by half in the $\triangle$ fur $\Delta r y h B$ strain $(\Delta$ fur $\Delta r y h B / \Delta$ fur $<0.5)$. However, the expression of $f h u A$ and sitA was significantly increased more than two-fold $(\Delta f u r \Delta r y h B / \Delta f u r>2.0)$. These results imply

\begin{tabular}{|c|c|c|}
\hline \multirow[t]{2}{*}{ Systems } & \multirow[t]{2}{*}{ Gene } & \multirow{2}{*}{$\begin{array}{l}\text { RNA expression ratio } \\
\Delta \text { fur } \Delta r y h B / \Delta f u r\end{array}$} \\
\hline & & \\
\hline \multicolumn{3}{|l|}{$\mathrm{Fe}^{3+}$} \\
\hline Ferrichrome & fhuA & $2.62 \pm 0.07$ \\
\hline Aerobactin & iucA & $0.19 \pm 0.06$ \\
\hline \multirow[t]{3}{*}{ Enterobactin } & fepA & $0.36 \pm 0.01$ \\
\hline & fepB & $0.33 \pm 0.05$ \\
\hline & entC & $0.46 \pm 0.02$ \\
\hline \multirow[t]{2}{*}{ Ferric citrate } & $f e c A$ & $0.19 \pm 0.02$ \\
\hline & fecE & $0.34 \pm 0.03$ \\
\hline Salmochelin & $i r o B$ & $0.52 \pm 0.05$ \\
\hline Heme & hmuR & $0.69 \pm 0.01$ \\
\hline \multicolumn{3}{|l|}{$\mathrm{Fe}^{2+}$} \\
\hline \multirow[t]{2}{*}{ Ferrous iron } & $f e o B$ & $0.55 \pm 0.18$ \\
\hline & sitA & $2.81 \pm 0.08$ \\
\hline
\end{tabular}

${ }^{\text {a }}$ Mean expression ratio $( \pm S D)$ of $\Delta f u r \Delta r y h B$ relative to $\Delta f u r$. that RyhB activates the expression of iucA, fepA, fepB, ent $C$, $f e c A$, and $f e c E$, but represses the expression of fhuA and sitA.

\section{Discussion}

In this study, we provide an initial characterisation of $K$. pneumoniae RyhB. In $K$. pneumoniae, sequence comparison indicated that the nucleotide sequence of the $r y h B$ gene (91 bp) is $92.3 \%$ identical to the $E$. coli version (90 bp). However, the promoter sequence of $K$. pneumoniae ryhB is only $72.4 \%$ identical to that of $E$. coli. In this study, we found that the expression of $r y h B$ in K. pneumoniae is directly repressed by Fur-Fe(II), as is the case in E. coli (Figure 1).

In addition, structure of the genomic neighbourhood of ryhB differs between the 2 species. In the E. coli genome, $r y h B$ is found between $y h h X$ and $y h h Y$. In the $K$. pneumoniae genome, ryhB is flanked by $y h h Y$ and a hypothetical ORF. By Pfam search, the hypothetical ORF was found to contain a bactofilin domain (E-value $=3.7$ e-24), which belongs to a new class of polymer-forming proteins that serve as versatile molecular scaffolds in a variety of cellular pathways [47]. Even though the function of this hypothetical protein in $K$. pneumoniae has not yet been investigated, we found that RyhB could strongly repress the expression of this hypothetical protein (unpublished data). This result suggests that RyhB could participate in a variety of cellular pathways in $K$. pneumoniae.

We previously showed in $K$. pneumoniae, Fur represses CPS biosynthesis via regulation of RmpA, RmpA2, and RcsA. In addition to these 3 regulators, one or more regulators may be involved in the Fur-mediated control of cps transcription [21]. In this study, we found that RyhB also participates in Fur-regulated CPS biosynthesis via activation of orf1 and orf 16 transcription and is independent of the 3 regulators, $\mathrm{RmpA}, \mathrm{RmpA} 2$, and RcsA (Figure 2 and 3). We want to further analyse whether any potential transcriptional regulator-binding motifs exist in the promoter sequences of orf1 and orf16. We noted that a binding site typical of IscR, a transcriptional repressor that controls $\mathrm{Fe}-\mathrm{S}$ biosynthesis [48], was located $172 \mathrm{bp}$ upstream of the translation start site of GalF (encoded by orf1, 5' -ATAACCTGAACGAAAA TAAGATTAT- $\left.3^{\prime}\right)$. The predication indicated that IscR could participate in control of orf1 expression. Furthermore, a previous study reported that RyhB promotes the degradation of iscSUA transcripts, resulting in an increase in the ratio of apo-IscR/holo-IscR [48]. Whether RyhB activates CPS biosynthesis via regulation of the ratio of apo-IscR/holo-IscR in K. pneumoniae awaits further analysis. However, the regulatory mechanism of cps transcription is more complex than expected; whether another unknown transcriptional regulator is involved in 
activation of RyhB's effect on orf16 transcription needs to be investigated. In addition, CPS is considered the major determinant that can protect the bacteria from phagocytosis and killing by serum factors $[8,9]$. In this study, higher serum resistance was found in $\Delta$ fur, but this higher serum resistance was attenuated by further deletion of $r y h B$ (Figure 4). We suggest the protective role of RyhB against serum killing is due to the activation of CPS biosynthesis.

In $E$. coli, RyhB plays a positive role in control of the intracellular iron concentration via the degradation of nonessential iron-using proteins or an increase in siderophore production [49-51]. In this study, we also found the deletion of $r y h B$ in $\Delta$ fur decreased siderophore production on the CAS plate under iron-limiting condition (Figure 5). Consistent with E. coli [51], RyhB in K. pneumoniae regulates siderophore production by activating the expression of enterobactin system genes (entC, fepA, and $f e p B$ ). In addition, we found that RyhB may activate $i u c A$ and $f e c A$ expression. Since sRNA may positively regulate its target mRNAs via an anti-antisense mechanism to disrupt an intrinsic inhibitory structure in the $5^{\prime}$ mRNA region that sequesters the ribosome-binding site and the first translation codon [52,53], the 5'untranslated regions of the iuc and $f e c$ operons were analysed for sequences complementary to RyhB by prediction with the bioinformatics application RNAhybrid [54] (http://bibiserv.techfak.uni-bielefeld.de/rnahybrid/ submission.html). However, no apparent base pairing was found in the $5^{\prime}$-untranslated region of the iuc or fec operons, suggesting that the activation of $i u c A$ and $f e c A$ by $\mathrm{RyhB}$ is not a result of direct interaction. Furthermore, RyhB was found to repress the expression of $f h u A$ and sit $A$ in $K$. pneumoniae. In $E$. coli, RyhB represses the expression of $f h u A$, which also corresponds to our results [35]. A possible paring between RyhB with the adjacent sequence of translational start site of $f h u A$ and sitA was also predicted by the RNAhybrid algorithm. Alignment of the protected residues predicts that RyhB forms a $7+4+4$ bp RNA duplex with the sitA mRNA (Additional file 1: Figure S1), but no apparent base pairing was found between RyhB and fhuA. However, the direct interaction of RyhB with the sitA mRNA remains to be confirmed. In $E$. coli, RyhB has been shown to repress several genes that are involved in ironbinding, which may increase the intracellular iron concentration, thereby allowing a better usage of iron and more complete Fur repression of these genes [35,55]. Nevertheless, this possibility in K. pneumoniae needs to be proven by careful experiments. In this study, the coordinated action of Fur and RyhB was found to regulate the expression of the iron acquisition systems for maintaining intracellular iron homeostasis in $K$. pneumoniae.

\section{Conclusions}

In this study, we provide an initial characterisation of $K$. pneumoniae RyhB. Our results suggest that RyhB plays an important role in the Fur regulon, which modulates the CPS biosynthesis and iron acquisition systems in $K$. pneumoniae, both of which contribute to the infectivity and survival of the bacterium.

\section{Methods}

\section{Bacterial strains, plasmids, and media}

Bacterial strains and plasmids used in this study are listed in Table 2. Primers used in this study are list in Additional file 2: Table S1. Bacterial were routinely cultured at $37^{\circ} \mathrm{C}$ in Luria-Bertani (LB) medium or M9 minimal medium supplemented with appropriate antibiotics. The antibiotics used include ampicillin $(100 \mu \mathrm{g} / \mathrm{ml})$, kanamycin $(25 \mu \mathrm{g} / \mathrm{ml})$, streptomycin $(500 \mu \mathrm{g} / \mathrm{ml})$, and tetracycline $(12.5 \mu \mathrm{g} / \mathrm{ml})$.

\section{Construction of the gene-deletion mutants}

Specific gene deletion was introduced into K. pneumoniae CG43S3 using an allelic exchange strategy as previously described [57]. The pKAS46 system was used in the selection of the mutants [59], and the mutations were respectively confirmed by PCR and Southern hybridization (data not shown).

\section{Measurement of promoter activity}

The promoter region of $r y h B$ was PCR-amplified with primer pair pGT44/pGT45, and the amplicons were then cloned into placZ15 [63]. The promoter-reporter plasmids, pRyhB15, pOrf12, pOrf315, and pOrf1617, were individually mobilized into $K$. pneumoniae strains by conjugation from $E$. coli S17-1 $\lambda$ pir. The bacteria were grown to logarithmic phase in LB broth with or without $200 \mu \mathrm{M}$ Dip $\left(\mathrm{OD}_{600}\right.$ of 0.7$)$, and the $\beta$ galactosidase activity was measured as previously described [63].

\section{EMSA}

Recombinant $K$. pneumoniae Fur protein was expressed in E. coli and purified as previously described [22]. DNA fragment of the putative promoter region of $r y h B$ was respectively PCR amplified by using specific primer sets (Table 2). The purified $\mathrm{His}_{6}$-Fur was incubated with 10ng DNA in a $15 \mu \mathrm{l}$ solution containing $50 \mathrm{mM}$ Tris- $\mathrm{HCl}$ (pH 7.5), $100 \mathrm{mM} \mathrm{NaCl}, 100 \mathrm{mM}$ dithiothreitol, $200 \mu \mathrm{M}$ $\mathrm{MnCl}_{2}$, and $1 \mu \mathrm{g} / \mu \mathrm{l}$ BSA at room temperature for $20 \mathrm{~min}$. The samples were then loaded onto a native gel of $5 \%$ nondenaturing polyacrylamide containing $5 \%$ glycerol in $0.5 \times$ TB buffer (45 mM Tris-HCl, pH 8.0, $45 \mathrm{mM}$ boric acid). Gels were electrophoresed with a 20-mA current at $4^{\circ} \mathrm{C}$ and then stained with SABR safe Gel stain (Invitrogen). 
Table 2 Bacterial strains and plasmids used in this study

\begin{tabular}{|c|c|c|}
\hline $\begin{array}{l}\text { Strains or } \\
\text { plasmids }\end{array}$ & Descriptions & Reference or source \\
\hline \multicolumn{3}{|l|}{ K. pneumoniae } \\
\hline CG43S3 & CG43 Sm ${ }^{r}$ & [56] \\
\hline$\Delta / a c Z$ & CG43S3 $\Delta$ lacZ & [17] \\
\hline$\Delta$ fur & CG43S3 $\Delta$ fur & [22] \\
\hline$\Delta / a c Z \Delta$ fur & CG43S3 3 lacZ $\Delta$ fur & {$[22]$} \\
\hline$\Delta r y h B$ & CG43S3 $\Delta r y h B$ & This study \\
\hline$\Delta f u r \Delta r y h B$ & CG43S3 $\Delta$ fur $\Delta r y h B$ & This study \\
\hline$\Delta / a c z \Delta f u r \Delta r y h B$ & CG43S3 $\Delta / a c z \Delta$ fur $\Delta r y h B$ & This study \\
\hline$\Delta$ galU & CG43S3 $\Delta$ galU & [57] \\
\hline \multicolumn{3}{|l|}{ E. coli } \\
\hline $\mathrm{DH} 5 \mathrm{a}$ & 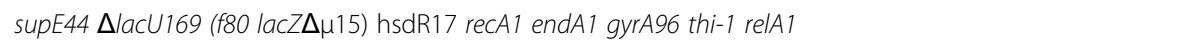 & [58] \\
\hline BL21-RIL & F ompT hsdS $S_{B}\left[r_{B}^{-} m_{B}^{-}\right] g a l d c m[D E 3]$ & Laboratory stock \\
\hline S17-1 $\lambda$ pir H1717 & 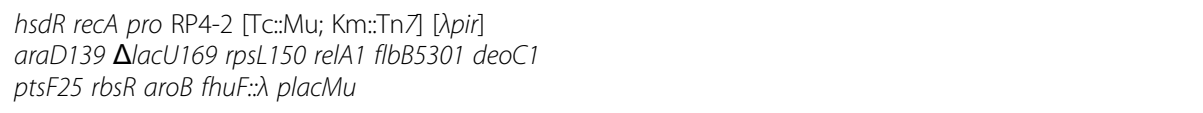 & {$[59,60]$} \\
\hline \multicolumn{3}{|l|}{ Plasmids } \\
\hline pKAS46 & Positive selection suicide vector, rps $\mathrm{Ap}^{r} \mathrm{Km}^{r}$ & [59] \\
\hline yT\&A & TA cloning vector & Yeastern \\
\hline pRK415 & Broad-host-range IncP cloning vector, $T c^{r}$ & [61] \\
\hline pT7-7 & Cloning vector, $A p^{r}$ & [62] \\
\hline pETQ & $\mathrm{Km}^{\mathrm{r}}$, protein expression vector & [61] \\
\hline placZ15 & $\mathrm{Cm}^{\mathrm{r}}$, promoter selection vector, lac $\mathrm{Z}^{+}$ & [17] \\
\hline pfur & $\mathrm{Tc}^{\mathrm{r}}, 0.8$-kb fragment containing a fur allele cloned into pRK415 & [22] \\
\hline pET30c-Fur & $\mathrm{Km}^{\mathrm{r}}$, 450-bp fragment encoding full-length Fur cloned into pET30c & [22] \\
\hline pRyhB04 & $2.0 \mathrm{~kb}$ fragment containing an internal $\sim 70-\mathrm{bp}$ deletion in ryhB cloned into pKAS46 & This study \\
\hline pRyhB15 & $\mathrm{Cm}^{r}$, 178-bp fragment containing the region upstream of ryhB cloned into placZ15 & This study \\
\hline pOrf12 & $\mathrm{Cm}^{r}$, 500-bp fragment containing the region upstream of Klebsiella K2 cps orf1-orf2 cloned into placZ15 & [17] \\
\hline pOrf315 & $\mathrm{Cm}^{r}$, 900-bp fragment containing the region upstream of Klebsiella K2 cps orf3-orf15 cloned into placZ15 & [17] \\
\hline pOrf1617 & $\mathrm{Cm}^{r}, 300$-bp fragment containing the region upstream of Klebsiella K2 cps orf16-orf17 cloned into placZ15 & [17] \\
\hline pT7-7-pryhB & 178-bp fragment containing the putative ryhB promoter, cloned into pT7-7 & This study \\
\hline pETQ-ryhB & $\mathrm{Km}^{\mathrm{r}}$, 326-bp fragment containing the promoter and coding region of ryhB cloned into pETQ & This study \\
\hline
\end{tabular}

\section{FURTA}

FURTA was performed according to the method described by Stojiljkovic et al. [64]. DNA sequences containing a putative Fur box were PCR amplified with specific primer sets and then cloned into pT7-7. The resulting plasmids were introduced into the $E$. coli strain H1717, and the transformants were plated onto MacConkey-lactose plates containing $100 \mu \mathrm{g} / \mathrm{ml}$ ampicillin and $30 \mu \mathrm{M} \mathrm{Fe}\left(\mathrm{NH}_{4}\right)_{2}\left(\mathrm{SO}_{4}\right)_{2}$. The indicator strain H1717 contained a chromosomal fhuF::lacZ fusion, and a low affinity Fur box has been demonstrated in the fhuF promoter. The introduction of pT7-7 derived plasmids carrying Fur-binding sequences could thus cause the removal of Fur from the fhuF Fur box [60]. H1717 harboring pT7-7 was used as a negative control. Colony phenotype was observed after incubation at $37^{\circ} \mathrm{C}$ for 10 h. Red colony (Lac+) denoted a FURTA-positive phenotype and indicated the binding of Fur to the DNA sequence cloned into the pT7-7 plasmid.

\section{Extraction and quantification of CPS}

CPS was extracted and quantified as previously described [65]. The glucuronic acid content, represents the amount of $K$. pneumoniae $\mathrm{K} 2 \mathrm{CPS}$, was determined from a standard curve of glucuronic acid (SigmaAldrich) and expressed as micrograms per $10^{9} \mathrm{CFU}$ [46].

\section{qRT-PCR}

Total RNAs were isolated from early-exponential-phase grown bacteria cells by use of the RNeasy midi-column 
(QIAGEN) according to the manufacturer's instructions. RNA was DNase-treated with RNase-free DNase I (MoBioPlus) to eliminate DNA contamination. RNA of $100 \mathrm{ng}$ was reverse-transcribed with the Transcriptor First Strand cDNA Synthesis Kit (Roche) using random primers. qRT-PCR was performed in a Roche LightCycler $^{\circledR} 1.5$ Instrument using LightCycler TaqMan Master (Roche). Primers and probes were designed for selected target sequences using Universal ProbeLibrary Assay Design Center (Roche-applied science) and listed in Additional file 2: Table S1. Data were analyzed using the real time PCR software of Roche LightCycler ${ }^{\circledR} 1.5$ Instrument. Relative gene expressions were quantified using the comparative threshold cycle $2^{-\Delta \Delta C T}$ method with 23S rRNA as the endogenous reference.

\section{Bacterial survival in serum}

Normal human serum, pooled from healthy volunteers, was divided into equal volumes and stored at $-70^{\circ} \mathrm{C}$ before use. Bacterial survival in serum was determined with minor modifications [57]. First, The bacteria were grown to log phase in LB broth and the viable bacterial concentration was adjusted to $1 \times 10^{6}$ colony forming units $/ \mathrm{ml} .1 \mathrm{ml}$ of the cultures was washed twice by using phosphate-buffered saline (PBS) and resuspended in $1 \mathrm{ml}$ PBS. The mixture containing $250 \mu \mathrm{l}$ of the cell suspension and $750 \mu \mathrm{l}$ of pooled human serum was incubated at $37^{\circ} \mathrm{C}$ for $60 \mathrm{~min}$. The number of viable bacteria was then determined by plate counting. The survival rate was expressed as the number of viable bacteria treated with human serum compared to the number of pretreatment. The assay was performed triple, each with triplicate samples. The data from one of the representative experiments are shown and expressed as the mean and standard deviation from the three samples. The $0 \%$ survival of $K$. pneumoniae CG43S3 $\Delta$ galU served as a negative control.

\section{CAS assay}

The CAS assay was performed according to the method described by Schwyn and Neilands [66]. Each of the bacterial strain was grown overnight in M9 minimal medium, and then $5 \mu$ of culture was added onto a CAS agar plate. After $24 \mathrm{hr}$ incubation at $37^{\circ} \mathrm{C}$, effects of the bacterial siderophore production could be observed. Siderophore production was apparent as an orange halo around the colonies; absence of a halo indicated the inability to produce siderophores.

\section{Statistical method}

An unpaired $t$-test was used to determine the statistical significance and values of $P<0.001$ were considered significant. The results of CPS quantification and qRTPCR analysis were derived from a single experiment representative of three independent experiments. Each sample was assayed in triplicate and the mean activity and standard deviation are presented.

\section{Additional files}

Additional file 1: Figure S1. RyhB pairs with sitA. The file contains supplemental figure $\mathrm{S} 1$ that the potential base pairing in RyhB/sitA mRNA in this study.

Additional file 2: Table S1. Primers used in this study. The file contains supplemental Table S1 that the detailed information of primer sets used in this study.

\section{Competing interests}

The authors declare that they have no competing interests.

\section{Acknowledgements}

The work is supported by the grants from National Science Council (NSC 972314-B-039-042-MY2 and NSC 99-2320-B-039-002-MY3) and China Medical University (CMU98-ASIA-01 and CMU99-ASIA-07).

\section{Author details}

${ }^{1}$ Department of Biotechnology, Asia University, Taichung 41354, Taiwan. ${ }^{2}$ Department of Biological Science and Technology, National Chiao Tung University, Hsin Chu 30068, Taiwan. ${ }^{3}$ Institute of Genomics and Bioinformatics, National Chung Hsing University, Tai Chung City 40227, Taiwan. ${ }^{4}$ Biotechnology Center, National Chung Hsing University, Tai Chung City 40227, Taiwan. ${ }^{5}$ Institute of Molecular and Genomic Medicine, National Health Research Institutes, Miaoli County 35053, Taiwan. ${ }^{6}$ School of Chinese Medicine, China Medical University, Taichung 40402, Taiwan.

\section{Authors' contributions}

$\mathrm{SHH}, \mathrm{CKW}, \mathrm{HLP}$, and CTL made substantial contributions to design and conduct the experiments. YMH performed qRT-PCR and growth experiments. $\mathrm{SHH}$ and $\mathrm{CKW}$ performed the bioinformatics analyses and interpretation of data. CCW, YTC, and HLP contributed to the writing and editing of the manuscript. CTL coordinated the study and performed manuscript editing. All authors have read and approved this work.

Received: 15 May 2012 Accepted: 9 July 2012

Published: 24 July 2012

\section{References}

1. Chou FF, Kou HK: Endogenous endophthalmitis associated with pyogenic hepatic abscess. J Am Coll Surg 1996, 182(1):33-36.

2. Han SH: Review of hepatic abscess from Klebsiella pneumoniae. An association with diabetes mellitus and septic endophthalmitis. West J Med 1995, 162(3):220-224.

3. Lau YJ, Hu BS, Wu WL, Lin YH, Chang HY, Shi ZY: Identification of a major cluster of Klebsiella pneumoniae isolates from patients with liver abscess in Taiwan. J Clin Microbiol 2000, 38(1):412-414

4. Peng HL, Wang PY, Wu JL, Chiu CT, Chang HY: Molecular epidemiology of Klebsiella pneumoniae. Zhonghua Min Guo Wei Sheng Wu Ji Mian Yi Xue Za Zhi 1991, 24(3):264-271.

5. Yang YS, Siu LK, Yeh KM, Fung CP, Huang SJ, Hung HC, Lin JC, Chang FY: Recurrent Klebsiella pneumoniae liver abscess: clinical and microbiological characteristics. J Clin Microbiol 2009, 47(10):3336-3339.

6. Paterson DL: Resistance in gram-negative bacteria: enterobacteriaceae. Am J Med 2006, 119(6 Suppl 1):S20-S28. discussion S62-70.

7. Lagamayo EN: Antimicrobial resistance in major pathogens of hospitalacquired pneumonia in Asian countries. Am J Infect Control 2008, 36(4 Suppl):S101-S108.

8. Sahly H, Podschun R, Oelschlaeger TA, Greiwe M, Parolis H, Hasty D, Kekow J, Ullmann U, Ofek I, Sela S: Capsule impedes adhesion to and invasion of epithelial cells by Klebsiella pneumoniae. Infect Immun 2000, 68(12):6744-6749.

9. Lin JC, Chang FY, Fung CP, XU JZ, Cheng HP, Wang JJ, Huang LY, Siu LK: High prevalence of phagocytic-resistant capsular serotypes of 
Klebsiella pneumoniae in liver abscess. Microbes Infect 2004, 6(13):1191-1198.

10. Boddicker JD, Anderson RA, Jagnow J, Clegg S: Signature-tagged mutagenesis of Klebsiella pneumoniae to identify genes that influence biofilm formation on extracellular matrix material. Infect Immun 2006, 74 (8):4590-4597.

11. Moranta D, Regueiro V, March C, Llobet E, Margareto J, Larrate E, Garmendia J, Bengoechea JA: Klebsiella pneumoniae capsule polysaccharide impedes the expression of beta-defensins by airway epithelial cells. Infect Immun 2010, 78(3):1135-1146.

12. Favre-Bonte S, Joly B, Forestier C: Consequences of reduction of Klebsiella pneumoniae capsule expression on interactions of this bacterium with epithelial cells. Infect Immun 1999, 67(2):554-561.

13. Fung CP, Hu BS, Chang FY, Lee SC, Kuo BI, Ho M, Siu LK, Liu CY: A 5-year study of the seroepidemiology of Klebsiella pneumoniae: high prevalence of capsular serotype $\mathrm{K} 1$ in Taiwan and implication for vaccine efficacy. $J$ Infect Dis 2000, 181(6):2075-2079.

14. Pan YJ, Fang HC, Yang HC, Lin TL, Hsieh PF, Tsai FC, Keynan Y, Wang JT: Capsular polysaccharide synthesis regions in Klebsiella pneumoniae serotype K57 and a new capsular serotype. J Clin Microbiol 2008, 46 (7):2231-2240.

15. Fung CP, Chang FY, Lee SC, Hu BS, Kuo BI, Liu CY, Ho M, Siu LK: A global emerging disease of Klebsiella pneumoniae liver abscess: is serotype $\mathrm{K} 1$ an important factor for complicated endophthalmitis? Gut 2002, 50 (3):420-424.

16. Arakawa $Y$, Wacharotayankun $R$, Nagatsuka T, Ito H, Kato N, Ohta M: Genomic organization of the Klebsiella pneumoniae cps region responsible for serotype K2 capsular polysaccharide synthesis in the virulent strain Chedid. J Bacteriol 1995, 177(7):1788-1796.

17. Lin CT, Huang TY, Liang WC, Peng HL: Homologous response regulators $\mathrm{KvgA}, \mathrm{KvhA}$ and KvhR regulate the synthesis of capsular polysaccharide in Klebsiella pneumoniae CG43 in a coordinated manner. J Biochem (Tokyo) 2006, 140(3):429-438

18. Majdalani N, Gottesman S: The Rcs phosphorelay: a complex signal transduction system. Annu Rev Microbiol 2005, 59:379-405.

19. Gottesman S, Stout V: Regulation of capsular polysaccharide synthesis in Escherichia coli K12. Mol Microbiol 1991, 5(7):1599-1606.

20. Stout $\mathrm{V}$ : Regulation of capsule synthesis includes interactions of the RcsC/RcsB regulatory pair. Res Microbiol 1994, 145(5-6):389-392.

21. Lin CT, Wu CC, Chen YS, Lai YC, Chi C, Lin JC, Chen Y, Peng HL: Fur regulation of the capsular polysaccharide biosynthesis and ironacquisition systems in Klebsiella pneumoniae CG43. Microbiology 2011, 157(Pt 2):419-429.

22. Cheng HY, Chen YS, Wu CY, Chang HY, Lai YC, Peng HL: RmpA regulation of capsular polysaccharide biosynthesis in Klebsiella pneumoniae CG43. J Bacteriol 2010, 192(12):3144-3158.

23. De Champs C, Sauvant MP, Chanal C, Sirot D, Gazuy N, Malhuret R, Baguet JC, Sirot J: Prospective survey of colonization and infection caused by expanded-spectrum-beta-lactamase-producing members of the family Enterobacteriaceae in an intensive care unit. J Clin Microbiol 1989, 27 (12):2887-2890.

24. Markowitz SM, Veazey JM Jr, Macrina FL, Mayhall CG, Lamb VA: Sequential outbreaks of infection due to Klebsiella pneumoniae in a neonatal intensive care unit: implication of a conjugative R plasmid. J Infect Dis 1980, 142(1):106-112

25. Ernst JF, Bennett RL, Rothfield LI: Constitutive expression of the ironenterochelin and ferrichrome uptake systems in a mutant strain of Salmonella typhimurium. J Bacteriol 1978, 135(3):928-934

26. Hantke K: Regulation of ferric iron transport in Escherichia coli K12: isolation of a constitutive mutant. Mol Gen Genet 1981, 182(2):288-292.

27. Achenbach $L A$, Yang $W$ : The fur gene from Klebsiella pneumoniae: characterization, genomic organization and phylogenetic analysis. Gene 1997, 185(2):201-207

28. Griggs DW, Konisky J: Mechanism for iron-regulated transcription of the Escherichia coli cir gene: metal-dependent binding of fur protein to the promoters. J Bacteriol 1989, 171(2):1048-1054.

29. Hassett DJ, Sokol PA, Howell ML, Ma JF, Schweizer HT, Ochsner U, Vasil ML: Ferric uptake regulator (Fur) mutants of Pseudomonas aeruginosa demonstrate defective siderophore-mediated iron uptake, altered aerobic growth, and decreased superoxide dismutase and catalase activities. J Bacteriol 1996, 178(14):3996-4003.
30. Ochsner UA, Vasil ML: Gene repression by the ferric uptake regulator in Pseudomonas aeruginosa: cycle selection of iron-regulated genes. Proc Natl Acad Sci USA 1996, 93(9):4409-4414

31. Bijlsma JJ, Waidner B, Vliet AH, Hughes NJ, Hag S, Bereswill S, Kelly DJ, Vandenbroucke-Grauls CM, Kist M, Kusters JG: The Helicobacter pylori homologue of the ferric uptake regulator is involved in acid resistance. Infect Immun 2002, 70(2):606-611.

32. van Vliet AH, Stoof J, Poppelaars SW, Bereswill S, Homuth G, Kist M, Kuipers EJ, Kusters JG: Differential regulation of amidase- and formamidasemediated ammonia production by the Helicobacter pylori fur repressor. J Biol Chem 2003, 278(11):9052-9057.

33. Wu CC, Lin CT, Cheng WY, Huang CJ, Wang ZC, Peng HL: Fur-dependent MrkHI regulation of type 3 fimbriae in Klebsiella pneumoniae CG43. Microbiology 2012, 158(Pt 4):1045-1056.

34. Hantke K: Iron and metal regulation in bacteria. Curr Opin Microbio/ 2001, 4(2):172-177.

35. Masse E, Vanderpool CK, Gottesman S: Effect of RyhB small RNA on global iron use in Escherichia coli. J Bacteriol 2005, 187(20):6962-6971.

36. Andrews SC, Harrison PM, Guest JR: Cloning, sequencing, and mapping of the bacterioferritin gene (bfr) of Escherichia coli K-12. J Bacteriol 1989, 171 (7):3940-3947.

37. Gruer MJ, Guest JR: Two genetically-distinct and differentially-regulated aconitases (AcnA and AcnB) in Escherichia coli. Microbiology 1994, 140(Pt 10):2531-2541

38. Niederhoffer EC, Naranjo CM, Bradley KL, Fee JA: Control of Escherichia coli superoxide dismutase ( $\operatorname{cod} A$ and $\operatorname{sod} B$ ) genes by the ferric uptake regulation (fur) locus. J Bacterio/ 1990, 172(4):1930-1938.

39. Masse $E$, Gottesman S: A small RNA regulates the expression of genes involved in iron metabolism in Escherichia coli. Proc Natl Acad Sci USA 2002, 99(7):4620-4625.

40. Masse E, Escorcia FE, Gottesman S: Coupled degradation of a small regulatory RNA and its mRNA targets in Escherichia coli. Genes Dev 2003, 17(19):2374-2383.

41. Dubrac S, Touati D: Fur positive regulation of iron superoxide dismutase in Escherichia coli: functional analysis of the sodB promoter. $J$ Bacteriol 2000, 182(13):3802-3808.

42. Davis BM, Quinones M, Pratt J, Ding Y, Waldor MK: Characterization of the small untranslated RNA RyhB and its regulon in Vibrio cholerae. J Bacteriol 2005, 187(12):4005-4014

43. Argaman L, Elgrably-Weiss M, Hershko T, Vogel J, Altuvia S: RelA protein stimulates the activity of RyhB small RNA by acting on RNA-binding protein Hfq. Proc Natl Acad Sci USA 2012, 109(12):4621-4626.

44. Mey AR, Craig SA, Payne SM: Characterization of Vibrio cholerae RyhB: the RyhB regulon and role of ryhB in biofilm formation. Infect Immun 2005, 73 (9):5706-5719.

45. Murphy ER, Payne SM: RyhB, an iron-responsive small RNA molecule, regulates Shigella dysenteriae virulence. Infect Immun 2007 , 75(7):3470-3477.

46. Blumenkrantz N, Asboe-Hansen G: New method for quantitative determination of uronic acids. Anal Biochem 1973, 54(2):484-489.

47. Kuhn J, Briegel A, Morschel E, Kahnt J, Leser K, Wick S, Jensen GJ, Thanbichler M: Bactofilins, a ubiquitous class of cytoskeletal proteins mediating polar localization of a cell wall synthase in Caulobacter crescentus. EMBO J 2010, 29(2):327-339.

48. Desnoyers G, Morissette A, Prevost K, Masse E: Small RNA-induced differential degradation of the polycistronic mRNA iscRSUA. EMBO J 2009, 28(11):1551-1561.

49. Masse E, Salvail H, Desnoyers G, Arguin M: Small RNAs controlling iron metabolism. Curr Opin Microbiol 2007, 10(2):140-145.

50. Jacques JF, Jang S, Prevost K, Desnoyers G, Desmarais M, Imlay J, Masse E: RyhB small RNA modulates the free intracellular iron pool and is essential for normal growth during iron limitation in Escherichia coli. Mol Microbiol 2006, 62(4):1181-1190

51. Salvail $H$, Lanthier-Bourbonnais $P$, Sobota JM, Caza M, Benjamin JA, Mendieta ME, Lepine F, Dozois CM, Imlay J, Masse E: A small RNA promotes siderophore production through transcriptional and metabolic remodeling. Proc Natl Acad Sci USA 2010, 107(34):15223-15228.

52. Frohlich KS, Vogel J: Activation of gene expression by small RNA. Curr Opin Microbiol 2009, 12(6):674-682.

53. Prevost K, Salvail H, Desnoyers G, Jacques JF, Phaneuf E, Masse E: The small RNA RyhB activates the translation of shiA mRNA encoding a permease 
of shikimate, a compound involved in siderophore synthesis. Mol Microbiol 2007, 64(5):1260-1273.

54. Rehmsmeier M, Steffen P, Hochsmann M, Giegerich R: Fast and effective prediction of microRNA/target duplexes. RNA 2004, 10(10):1507-1517.

55. Salvail $H$, Masse E: Regulating iron storage and metabolism with RNA: an overview of posttranscriptional controls of intracellular iron homeostasis. Wiley Interdiscip Rev RNA 2012, 3(1):26-36.

56. Lai $Y C$, Peng HL, Chang HY: Identification of genes induced in vivo during Klebsiella pneumoniae CG43 infection. Infect Immun 2001, 69(11):7140-7145.

57. Lai YC, Peng HL, Chang HY: RmpA2, an activator of capsule biosynthesis in Klebsiella pneumoniae CG43, regulates K2 cps gene expression at the transcriptional level. J Bacteriol 2003, 185(3):788-800.

58. Hanahan D: Studies on transformation of Escherichia coli with plasmids. J Mol Biol 1983, 166(4):557-580.

59. Skorupski K, Taylor RK: Positive selection vectors for allelic exchange. Gene 1996, 169(1):47-52.

60. Hantke K: Selection procedure for deregulated iron transport mutants (fur) in Escherichia coli K 12: fur not only affects iron metabolism. Mol Gen Genet 1987, 210(1):135-139.

61. Keen NT, Tamaki S, Kobayashi D, Trollinger D: Improved broad-host-range plasmids for DNA cloning in gram-negative bacteria. Gene 1988, 70 (1):191-197.

62. Tabor S, Richardson CC: A bacteriophage T7 RNA polymerase/promoter system for controlled exclusive expression of specific genes. Proc Nat Acad Sci USA 1985, 82(4):1074-1078.

63. Lin CT, Huang TY, Liang WC, Peng HL: Homologous response regulators KvgA, KvhA and KvhR regulate the synthesis of capsular polysaccharide in Klebsiella pneumoniae CG43 in a coordinated manner. J Biochem 2006, 140(3):429-438.

64. Stojiljkovic I, Baumler AJ, Hantke K: Fur regulon in gram-negative bacteria. Identification and characterization of new iron-regulated Escherichia coli genes by a fur titration assay. J Mol Biol 1994, 236(2):531-545.

65. Domenico P, Schwartz S, Cunha BA: Reduction of capsular polysaccharide production in Klebsiella pneumoniae by sodium salicylate. Infect Immun 1989, 57(12):3778-3782.

66. Schwyn B, Neilands JB: Universal chemical assay for the detection and determination of siderophores. Anal Biochem 1987, 160(1):47-56.

doi:10.1186/1471-2180-12-148

Cite this article as: Huang et al:: Role of the small RNA RyhB in the Fur regulon in mediating the capsular polysaccharide biosynthesis and iron acquisition systems in Klebsiella pneumoniae. BMC Microbiology 2012

$12: 148$

\section{Submit your next manuscript to BioMed Central and take full advantage of:}

- Convenient online submission

- Thorough peer review

- No space constraints or color figure charges

- Immediate publication on acceptance

- Inclusion in PubMed, CAS, Scopus and Google Scholar

- Research which is freely available for redistribution 